\title{
DOPPLER COMPENSATION IN UNDERWATER CHANNELS USING TIME-REVERSAL ARRAYS
}

\author{
João Gomes \\ Victor Barroso \\ Instituto Superior Técnico - Instituto de Sistemas e Robótica \\ Av. Rovisco Pais, 1049-001 Lisboa, Portugal \\ $\{$ jpg,vab\}@isr.ist.utl.pt
}

\begin{abstract}
This work addresses the problem of compensating for differential Doppler shifts due to source motion in a time-reversal mirror. Time reversal is a feedback wave focusing technique that can be used in poorly-characterized media, and is quite appealing in bidirectional underwater communications as it allows multipath to be transparently canceled in highly dispersive channels. Under simplifying assumptions, it is shown that time-reversed focusing occurs even when the source moves uniformly, although this induces fluctuations in the envelope of the regenerated signal due to differential Doppler. The proposed technique for Doppler compensation builds on the representation of time-varying pulse shapes using delay-Doppler spread functions. By inverting these functions in both delay and Doppler at each transducer, the mirror is able to project signals that converge on the source and are devoid of Doppler shifts when observed in its moving reference frame.
\end{abstract}

\section{INTRODUCTION}

Much effort has been devoted to the development of underwater acoustic communication systems that can cope with challenging environmental conditions, such as severe multipath due to sound reflection on the surface and bottom. Recently, time reversal has been explored as a complement to conventional multipath compensation based on adaptive equalization $[1,2]$. Time reversal is a generic wave focusing technique that exploits the reciprocity of wave propagation in linear media to concentrate energy at a given focal spot with no specific information about the environment. Practical time-reversal in the ocean relies on an array formed by several source-receiver transducers that sample the incoming pressure field generated by an acoustic source $[3,4]$. When the received signals are played back in reverse, they interfere to create a reciprocal field that converges on the original source position. All multipath replicas are thus synchronously combined at the focus, resulting in waveforms with low delay dispersion. In analogy with the reflection of light in a mirror, this type of array is known as a time-reversal mirror (TRM).

These self-focusing properties immediately suggest applications in underwater communication, with the TRM acting as a transmitter-side device that cancels multipath at the focus. The residual interference may be sufficiently low so that only minimal equalization is subsequently needed to attain low bit error rates

This work was partially supported by the FCT Programa Operacional Sociedade da Informação (POSI) in the frame of QCA III, under contract PCTI/1999/CPS/33205.
[1]. Additionally, the interference of multiple synchronized replicas tends to favor the stability of pressure fields at the focus, thus mitigating the effects of channel fading.

In active phase conjugation for coherent communication, templates of the distorted signaling pulses are stored at the array during an initial (forward) transmission originating at the focus. These waveforms are time-reversed at the mirror and modulated by a message sequence during the so-called reciprocal phase to regenerate a clean PAM signal at the focus. The medium must remain reasonably stable between the forward and reciprocal transmissions for this strategy to be effective. However, one should note that the focusing effect is preserved even in the presence of some types of nonstationarity. As demonstrated theoretically in [3] for a moving source and an ideal mirror, focusing still occurs along the timereversed trajectory if the surrounding environment remains static. This property is of considerable practical interest, e.g., when communicating with underwater vehicles.

In this paper, strategies for dealing with Doppler due to source motion are investigated. It is shown that Doppler basically induces a uniform rotation of otherwise time-invariant path responses, causing undesirable envelope modulation in communication signals. A technique is developed for changing the Doppler shifts associated with distinct propagation rays that impinge upon the TRM without destroying the sensitive focusing information that is implicitly contained in the received pulse shapes. This allows automatic compensation of differential Doppler at the receiver during the time-reversed transmission even if, as would most likely happen in a real situation, it is not following the time-reversed path. This simple and robust approach can be combined with other variants of time reversal, such as spatial modulation through wavefront segmentation $[2,5]$.

\section{IDEAL TIME-REVERSAL CAVITY}

This section summarizes some background results on phase conjugation that are relevant to the work presented here. See, e.g., [3] for a much more thorough presentation.

The propagation of a pressure wave generated by a point source in a non-dispersive medium is governed by the wave equation. Its solutions are time reversible in the sense that the equation is still satisfied if the time variable is inverted. The required boundary conditions for the time-reversed field may be specified through the Helmholtz-Kirchoff integral, which relates the field at a given point in terms of an integral over an arbitrary closed surface surrounding that point. In the case of a monochromatic point source, if the conjugates of the measured field and its gradient are imposed 
as boundary conditions along that surface, then the resulting complex pressure inside this time-reversal cavity does not depend on the surface shape [3]. It is given by the real part of the original (free-space) field, implying that large pressure values will be observed in the vicinity of the source location. The argument may be extended to demonstrate that the time-dependence of a broadband point source will be approximately regenerated at the focus, reversed in time.

Similarly to the static case, a source with arbitrary space-time dependence $\tilde{x}(\mathbf{r}, t)$ may be harmonically decomposed into a superposition of monochromatic point sources. For uniform motion $\tilde{x}(\mathbf{r}, t)=\tilde{x}(t) \delta\left(\mathbf{r}-\mathbf{r}_{0}-\mathbf{v} t\right)$, the time-reversed field at $\mathbf{r}$ has the form [3]

$$
\tilde{z}(\mathbf{r}, t)=\frac{\tilde{x}\left(-t+\tau_{-}\right)-\tilde{x}\left(-t+\tau_{+}\right)}{R(-t) d(t)},
$$

where $\mathbf{v}$ is the constant source velocity vector, and $R(t)=\| \mathbf{r}-$ $\mathbf{r}_{0}-\mathbf{v} t \|$ is the distance between the source and field point at time $t$. The exact expressions for $\tau_{ \pm}$and $d(t)$ are omitted, as they have little relevance to this discussion. From (1) it can be concluded that, in the presence of a uniformly-moving point source, large pressure values are only obtained when $R(-t) \approx 0$, i.e., along the time-reversed trajectory. As in a static setting, the timedependence along that path is given by the time-reversed derivative of $\tilde{x}(t)$, which is essentially a scaled and reversed version of the original transmission for the type of passband signals that are assumed here.

In actual ocean experiments the ideal time-reversal (closed) surface is typically replaced by a linear vertical array of discrete sensors that only operate on the pressure field, not on its gradient. Even with this coarse approximation, it is possible to demonstrate theoretically and experimentally that a strong focus is still created at the expected position $[4,1]$.

\section{COHERENT COMMUNICATION IN THE PRESENCE OF DOPPLER}

As usually done in the context of communications, a complex representation in terms of lowpass equivalent signals will henceforth be adopted. Let $q(t)$ represent the basic pulse shape of the PAM sequences that are exchanged between the source and the TRM. Denoting by $g_{m}(t)$ the impulse response between a fixed (focal) point and the $m$-th sensor of an array with $M$ elements, multipath self-compensation implies that [2]

$$
q(t) *\left(\sum_{m=1}^{M} g_{m}(t) * g_{m}^{*}(-t)\right) \approx C q(t),
$$

where $C$ is a constant and $*$ is the convolution operator. It is assumed that the delay between the forward and time-reversed transmissions is sufficiently small so that the source displacement during that interval will be negligible, and the multipath structure of the channel can be regarded as constant [5]. Fluctuations in the phase of $g_{m}(t)$ due to uniform motion will simply be lumped together with actual Doppler shifts for estimation/compensation purposes, as described below.

The equivalent Doppler-distorted signal transmitted over a single path in response to the nominal passband waveform $\tilde{x}(t)=$ $\operatorname{Re}\left\{x(t) e^{j \omega_{c} t}\right\}$ is [5]

$$
\tilde{x}\left(t\left(1+\beta_{S}\right)\right) \approx \operatorname{Re}\left\{x^{\prime}(t) e^{j \omega_{c} t}\right\}, \quad x^{\prime}(t)=x(t) e^{j \nu t},
$$

where $\beta_{S}$ is the time compression factor and $\nu=\omega_{c} \beta_{S}$. For a PAM signal generated from the complex symbol sequence $\{a(k)\}$ with signaling interval $T_{b}$, convolution of (3) with the impulse response of the $p$-th path, $g_{m, p}(t)$, yields the contribution at the $m$-th sensor

$$
\begin{aligned}
& y_{m, p}(t)=\int g_{m, p}(\tau) x(t-\tau) e^{j \nu_{m, p}(t-\tau)} d \tau \\
& =\sum_{k} a(k) e^{j \nu_{m, p} k T_{b}} h_{m, p}\left(t-k T_{b}\right), \\
& h_{m, p}(t) \triangleq q(t) e^{j \nu_{m, p} t} * g_{m, p}(t) .
\end{aligned}
$$

From the form of (4)-(5) it is seen that the contribution of each path can still be regarded as a PAM sequence with distorted pulse shape $h_{m, p}(t)$, but with a constellation $a^{\prime}(k)=a(k) e^{j \nu_{m, p} k T_{b}}$ that rotates from symbol to symbol.

To gain insight into the properties of the time-reversed signal, assume that there are $P$ path contributions for each sensor and the Doppler shifts for a given path are approximately equal across all array sensors, $\nu_{m, p} \approx \bar{\nu}_{p}$. If the TRM simply records the received signals $y_{m}(t)=\sum_{p=1}^{P} y_{m, p}(t)$, conjugates and plays them back in reverse, then the signal at the focus is given by

$$
\begin{aligned}
z(t)= & \sum_{m=1}^{M} g_{m}(t) * y_{m}^{*}(-t)=\sum_{k} a^{*}(-k) \\
& \cdot \sum_{p=1}^{P} e^{j \bar{\nu}_{p} k T_{b}} \sum_{m=1}^{M}\left[g_{m}(t) * h_{m, p}^{*}(-t)\right]_{t-k T_{b}} .
\end{aligned}
$$

But from (2) and (5) the inner summation in (6) reduces to

$$
q^{*}(-t) e^{j \bar{\nu}_{p} t} *\left(\sum_{m=1}^{M} g_{m}(t) * g_{m, p}^{*}(-t)\right) \approx C_{p} e^{j \bar{\nu}_{p} t} q^{*}(-t) .
$$

This is a consequence of the spatial filtering properties of timereversal, since the sum of impulse response convolutions in (7) can be interpreted as the response of a broadband beamformer matched to the parameters of the $p$-th wavefront to a superposition of $P$ wavefronts that make up $\left\{g_{m}(t)\right\}_{m=1}^{M}$. As discussed in [2], when the spatial selectivity is high the dominant term is $\sum_{m} g_{m, p}(t) *$ $g_{m, p}^{*}(-t) \approx C_{p} \delta(t)$. Substituting (7) in (6) finally yields

$$
z(t)=\left(\sum_{p=1}^{P} C_{p} e^{j \bar{\nu}_{p} t}\right) \cdot \sum_{k} a^{*}(-k) q^{*}\left(-\left(t-k T_{b}\right)\right) .
$$

This PAM signal, which does not suffer from delay dispersion, is an instantaneous sum of $P$ paths, each having its own Doppler shift. Large-scale envelope variations in (8) are caused by timevarying interference among these contributions.

\section{DOPPLER COMPENSATION}

According to (8), a static observer at the focus perceives the same Doppler shifts induced at the TRM by a source with constant velocity $\mathbf{v}$. If, however, the observer were moving uniformly along $-\mathbf{v}$, the original shifts would be precisely compensated in its reference frame, and the original (time-reversed) PAM waveform would be recovered. By contrast, the moving receiver observes a multipath-free signal with twice the original Doppler shift on each propagation path if the velocity is kept constant. In order to simplify the 
receiver, it would be desirable to synthetically change the Doppler shifts by processing at the TRM, while retaining the focusing information contained in the forward-transmitted replicas.

Due to the particular structure of Doppler-induced variations implied by (4)-(5) it should be possible to decouple the estimation of pulse components along the delay and Doppler axis. This property stems from the assumption that path impulse responses remain approximately static even as the source moves but, as shown in Section 2, is not really required for wave focusing. For increased robustness, and in keeping with the general philosophy of time reversal as a means of handling coarsely-modeled environments, signaling pulses are estimated as generic time-variant functions. To this end, use (4)-(5) to write

$$
\begin{aligned}
y_{m}(t) & =\sum_{k} a(k) h_{m}\left(k T_{b}, t-k T_{b}\right) \\
h_{m}(t, \tau) & =\sum_{p=1}^{P} e^{j \nu_{m}, p t} h_{m, p}(\tau),
\end{aligned}
$$

Rather than estimating (10) directly, its Fourier transform along the $t$ axis (delay-Doppler spread function [6]) is considered.

$$
U_{m}(\tau, \nu)=F_{t}\left\{h_{m}(t, \tau)\right\} \triangleq \int h_{m}(t, \tau) e^{-j \nu t} d t,
$$

Reflecting the structure of propagation in multiple paths, ocean impulse responses are often sparse in $\tau$. Given the exponential nature of time variations in $t$, this implies that $U_{m}(\tau, \nu)$ is sparse in both its arguments, i.e., it basically consists of a series of wellspaced peaks that are associated with the various paths. This fact can be used to drastically reduce the number of parameters needed for channel estimation and tracking [7].

As shown by (8), simple time-reversal of the received signals $y_{m}^{*}(-t)=\sum_{k} a^{*}(-k) h_{m}^{*}\left(-k T_{b},-\left(t-k T_{b}\right)\right)$ leads to residual rotation of path contributions at the focus. Instead of implicitly using $U_{m}^{*}(-\tau, \nu)=F_{t}\left\{h_{m}^{*}(-t,-\tau)\right\}$ to synthesize PAM waveforms, suppose now that $U_{m}(\tau, \nu)$ is estimated at the TRM and inverted in $\nu$ before generating the signal

$$
\begin{aligned}
x_{m}(t) & =\sum_{k} a^{*}(-k) \int U_{m}^{*}\left(-\left(t-k T_{b}\right),-\nu\right) e^{j \nu k T_{b}} \frac{d \nu}{2 \pi} \\
& =\sum_{k} a^{*}(-k) \sum_{p=1}^{P} e^{-j \nu_{m, p} k T_{b}} h_{m, p}^{*}\left(-\left(t-k T_{b}\right)\right) .
\end{aligned}
$$

The approximation (7) can still be invoked to write the modified focused signal $z(t)=\sum_{m} g_{m}(t) * x_{m}(t)$ as

$$
z(t)=\sum_{k} a^{*}(-k) q^{*}\left(-\left(t-k T_{b}\right)\right) \sum_{p=1}^{P} C_{p} e^{j \bar{\nu}_{p}\left(t-2 k T_{b}\right)}
$$

But the original PAM pulse $q(t)$ has an effective time span of only a few symbol intervals, hence each $k, p$ term in (13) will only take on significant values in a time window $(k \pm \Delta) T_{b}$. Under all plausible conditions $\left|\bar{\nu}_{p} T_{b} \Delta\right| \ll 1$, and $e^{j \bar{\nu}_{p}\left(t-2 k T_{b}\right)} \approx e^{-j \bar{\nu}_{p} k T_{b}} \approx$ $e^{-j \bar{\nu}_{p} t}$. Then (13) coincides with (8), except that the Doppler shifts are inverted. If the receiver keeps moving with velocity $\mathbf{v}$ during the reciprocal transmission phase, then it will observe a multipath-free and Doppler-free signal.
In practice $U_{m}$ would be estimated at the TRM from a (deterministic) time-frequency crosscorrelation between the forwardtransmitted signal, $x(t)$, and the received replica at the $m$-th transducer

$$
\hat{U}_{m}(\tau, \nu)=\int_{-\infty}^{\infty} y_{m}(t)\left\{x(t-\tau) e^{j \nu t}\right\}^{*} d t
$$

In fact, it can be shown that the estimate (14) equals the bidimensional convolution between the desired delay-Doppler spread function and the time-frequency autocorrelation of the transmitted signal. Ideally, the latter should equal $\delta(\tau) \delta(\nu)$, so that $\hat{U}_{m}(\tau, \nu)=$ $U_{m}(\tau, \nu)$. This goal can be approximately attained by properly parametrized finite-length PAM sequences [7], which are particularly well-suited to the present context. Under those conditions, the Doppler resolution is the reciprocal of the effective time span of $x(t)$, and for the scenarios of interest this would lead to a training signal lasting for about 5-10 seconds. This range of values is somewhat larger than usual in underwater telemetry, but not unreasonably so.

For signals having an essentially finite span along the delay and Doppler axes, the continuous delay-Doppler spread function and the time-frequency crosscorrelation can be discretized on a 2D grid with negligible information loss. These issues are considered in detail in [6], and justify writing a discrete-time input-output relation in terms of $U_{m}(k, l)=\Delta \tau \Delta \nu U_{m}(k \Delta \tau, l \Delta \nu)$.

\section{SIMULATION RESULTS}

The simulated environment is a range-independent ocean crosssection with $130 \mathrm{~m}$ depth. The source is located at a depth of $70 \mathrm{~m}$ and a range of $2 \mathrm{Km}$ from the TRM. Square-root raised cosine pulses with $20 \%$ rolloff were transmitted at $2 \mathrm{Kbaud}$, with $10 \mathrm{KHz}$ carrier frequency. Acoustic propagation was simulated using a Gaussian beam ray tracer with sound-speed profile shown in Figure 1a, constant bottom reflection coefficient $\alpha_{B}=0.6$, and surface reflection modeled as a deterministic angle-dependent coefficient equal to the average specular component for a surface RMS roughness of $0.4 \mathrm{~m}$ [7]. The source velocity vector was chosen as $\mathbf{v}=\left(\frac{\sqrt{2}}{2}, \frac{\sqrt{2}}{2}\right) \mathrm{m} / \mathrm{s}$, leading to differential Doppler shifts between rays with the same number of surface or bottom bounces of up to about $1.5 \mathrm{~Hz}$.

Figures 1b-c show two projections of the depth-delay-Doppler spread function, calculated according to (5), (10) and (11), and discretized with steps $\Delta \tau=T_{b} / 4$ and $\Delta \nu=0.1 \mathrm{~Hz}$. These projections correspond to $\sqrt{\sum_{l}\left|U_{m}(k, l)\right|^{2}}$ and $\sqrt{\sum_{m}\left|U_{m}(k, l)\right|^{2}}$, respectively. The multipath arrival structure, with surface- and bottom-reflected wavefronts impinging upon the array, is clearly visible on Figure 1b. It is also clear that $U_{m}(k, l)$ is indeed sparse, as most of the energy is concentrated around a one-dimensional trajectory traced by the ray arrivals as a function of depth, delay and Doppler.

According to the basic time-reversal strategy of Section 3, PAM sequences were generated at the mirror with time-varying pulse shapes described by $U_{m}^{*}(-k, l)$. Figure 2 shows the delayDoppler spread function at the focus for a TRM with $M=520$ uniformly-spaced sensors spanning the water column. This large number of sensors was chosen as a good approximation to an ideal continuous mirror, to avoid the problems associated with grating lobes due to spatial undersampling that are not relevant here.

In agreeement with (8), it can be seen that multipath has been virtually eliminated and the resulting pulse has negligible delay 


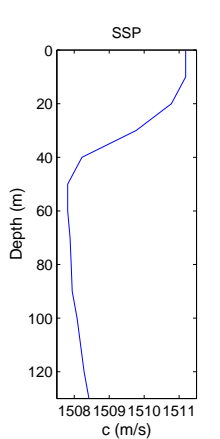

(a)

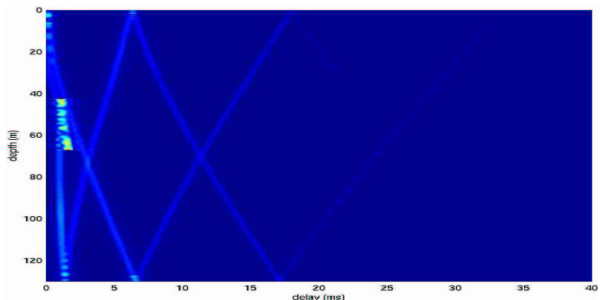

(b)

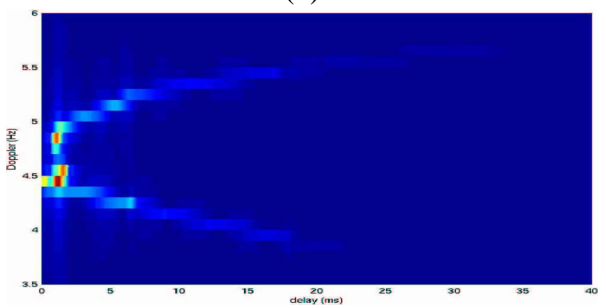

(c)

Fig. 1. (a) Sound-speed profile (b) Depth-delay projection of discretized depth-delay-Doppler spread function (c) Delay-Doppler projection

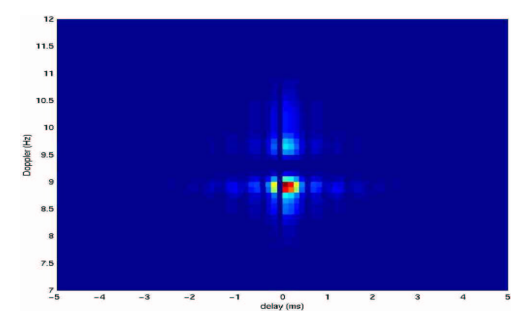

(a)

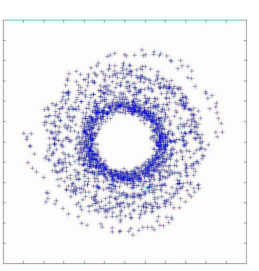

(b)
Fig. 2. Performance of plain TRM at moving receiver (a) DelayDoppler spread function (b) 2-PSK constellation

dispersion. Notice that the delay-Doppler spread function was calculated in the reference frame of the moving source/focus, hence the Doppler shifts for the $P$ replicas are located at twice the frequency values that appear in (8). Also shown in Figure 2 is the 2-PSK constellation at the receiver after root raised-cosine filtering of the PAM signal. In addition to circular rotation, there is significant magnitude modulation that results from time-varying interference between arrivals with different Doppler shifts over a period of $1 \mathrm{~s}$.

Figure 3 shows simulation results when the Doppler compensation procedure of section 4 is used, which simply amounts to inverting the Doppler index $U_{m}^{*}(-n, l) \rightarrow U_{m}^{*}(-n,-l)$. Similarly to Figure 2 there is negligible multipath, but now compression has been achieved in the Doppler axis as well, resulting in an impulselike spread function centered at $(0,0)$. The constellation confirms that both intersymbol interference (ISI) and magnitude distortion are weak, making the PAM signal easily decodable with simple receiver structures.

\section{CONCLUSION}

An approach for handling Doppler shifts in coherent time-reversed underwater communications was proposed. Under the simplifying

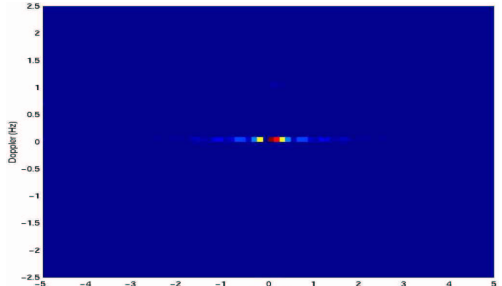

(a)

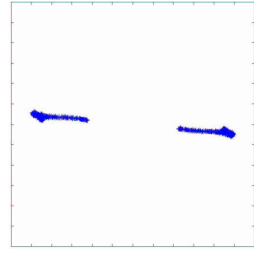

(b)
Fig. 3. Performance of Doppler-compensated TRM at moving receiver (a) Delay-Doppler spread function (b) 2-PSK constellation

assumptions of uniform source motion and invariant medium impulse responses, expressions were derived for the signals received at the focal spot, showing that multipath compensation is achieved even in the presence of differential Doppler, although the signal constellation suffers from residual rotation and magnitude modulation. A transmitter-side compensation method was proposed to change the Doppler shifts without affecting the focusing information gathered at the mirror. The moving receiver can therefore experience fully static conditions, and demodulate data packets with simplified processing blocks.

This method is conceptually very simple and conforms to the general emphasis of time reversal as a robust wave focusing technique that can be used in poorly-characterized linear media. However, manipulating depth-delay-Doppler spread functions on a 3D grid is computationally intensive and the overall accuracy can be affected by the accumulation of estimation errors. Developing efficient representations and estimation/tracking techniques that exploit the sparseness of such functions is one of the possible topics for future work.

\section{REFERENCES}

[1] G. Edelmann et al., "Underwater acoustic communication using time reversal," in Proc. of MTS/IEEE OCEANS'01, vol. 4, pp. 2231-2235, Honolulu, Hawaii, Nov. 2001.

[2] J. Gomes, V. Barroso, "Wavefront segmentation in phaseconjugate arrays for spatially modulated acoustic communication," in Proc. of MTS/IEEE OCEANS'01, vol. 4, pp. 22362243, Honolulu, Hawaii, Nov. 2001.

[3] D. Jackson, D. Dowling, "Phase conjugation in underwater acoustics," J. Acoust. Soc. Am., vol. 89, no. 1, pp. 171-181, Jan. 1991.

[4] W. Kuperman et al., "Phase conjugation in the ocean: Experimental demonstration of an acoustic time-reversal mirror," $J$. Acoust. Soc. Am., vol. 103, no. 1, pp. 25-40, Jan. 1998.

[5] J. Gomes, V. Barroso, "Time-reversed communication over Doppler-spread underwater channels," in Proc. of ICASSP'02, vol. 3, pp. 2849-2852, Orlando, FL, May 2002.

[6] P. Bello, "Characterization of randomly time-variant linear channels," IEEE Trans. on Comm. Systems, vol. CS-11, pp. 360-393, Dec. 1963.

[7] T. Eggen, Underwater Acoustic Communication over Doppler Spread Channels, Ph.D. thesis, MIT, Jun. 1997. 